\title{
Broad application of a simple and affordable protocol for isolating plant RNA
}

\author{
Daniel Couto', Lena Stransfeld ${ }^{1}$, Ana Arruabarrena², Cyril Zipfel ${ }^{1 *}$ and Rosa Lozano-Durán ${ }^{1,3^{*}}$
}

\begin{abstract}
Background: Standard molecular biological methods involve the analysis of gene expression in living organisms under diverse environmental and developmental conditions. One of the most direct approaches to quantify gene expression is the isolation of RNA. Most techniques used to quantify gene expression require the isolation of RNA, usually from a large number of samples. While most published protocols, including those for commercial reagents, are either labour intensive, use hazardous chemicals and/or are costly, a previously published protocol for RNA isolation in Arabidopsis thaliana yields high amounts of good quality RNA in a simple, safe and inexpensive manner.

Findings: We have tested this protocol in tomato and wheat leaves, as well as in Arabidopsis leaves, and compared the resulting RNA to that obtained using a commercial phenol-based reagent. Our results demonstrate that this protocol is applicable to other plant species, including monocots, and offers yield and purity at least comparable to those provided by commercial phenol-based reagents.

Conclusions: Here, we show that this previously published RNA isolation protocol can be easily extended to other plant species without further modification. Due to its simplicity and the use of inexpensive reagents, this protocol is accessible and affordable and can be easily implemented to work on different plant species in laboratories worldwide.
\end{abstract}

Keywords: RNA, Arabidopsis, Tomato, Wheat, Affordable, Protocol

\section{Findings}

The isolation of good quality RNA in sufficient amounts is often a prerequisite for the analysis of gene expression, an approach that is widely used in laboratories worldwide routinely. However, most published protocols are labour intensive and/or use hazardous chemicals, and commercially available reagents or kits usually use toxic chemicals and are costly [1-3]. These constrictions can make RNA isolation a bottleneck for gene expression analysis in many laboratories, especially when involving large numbers of samples.

Oñate-Sánchez \& Vicente-Carbajosa published an improved RNA isolation protocol for Arabidopsis that is simple, efficient, affordable, and avoids the use of toxic volatiles [4]. This article has been highly accessed, and

\footnotetext{
*Correspondence: cyril.zipfel@tsl.ac.uk; lozano-duran@sibs.ac.cn ${ }^{1} T$ he Sainsbury Laboratory, Norwich Research Park, Norwich NR4 7UH, United Kingdom

${ }^{3}$ Shanghai Institutes of Biological Sciences, Shanghai Center for Plant Stress Biology (PSC), Chinese Academy of Sciences, Shanghai 201602, China

Full list of author information is available at the end of the article
}

the protocol successfully implemented in laboratories working on this model plant. However, a growing body of plant biologists work on other model or non-model species, including crops, and therefore information about the wide applicability of this or a similar protocol would be highly beneficial to a large number of researchers.

We set out to determine (i) whether the RNA isolation protocol described by Oñate-Sánchez and VicenteCarbajosa [4] (see Additional file 1) can be applied to other plant species, and (ii) how the performance of this protocol compares to that of commercial phenol-based ready-to-use reagents. With this aim, we systematically extracted RNA from (a) Arabidopsis rosette leaves, (b) wheat leaves and (c) tomato leaves, using the protocol by Oñate-Sánchez and Vicente-Carbajosa [4], which uses non-toxic buffers containing sodium citrate and citric acid, and a commercial phenol-based reagent (TRI reagent, SIGMA, following the manufacturer's instructions) in parallel. The resulting RNA samples were subjected to spectrophotometric quantification using nanodrop (NanoDrop 8000, Thermo Scientific), and the RNA absorbance ratios $\left(\mathrm{A}_{260 / \mathrm{A} 230}\right.$ and $\left.\mathrm{A}_{260 / \mathrm{A} 280}\right)$ 
Table 1 Spectrophotometric determination of total RNA quantity and quality

\begin{tabular}{|c|c|c|c|c|c|c|}
\hline \multirow[b]{2}{*}{ Samples } & \multicolumn{3}{|l|}{ TRI reagent } & \multicolumn{3}{|c|}{ Oñate-Sánchez and Vicente-Carbajosa, 2008} \\
\hline & Concentration (ng/ $\mu \mathrm{L})$ & A260/280 & A260/230 & Concentration $(\mathrm{ng} / \mu \mathrm{L})$ & $A 260 / 280$ & A260/230 \\
\hline Arabidopsis 1 & 355.2 & 2.12 & 1.91 & 613.3 & 2.11 & 2.36 \\
\hline Arabidopsis 2 & 497.4 & 2.00 & 1.90 & 501.6 & 2.03 & 2.19 \\
\hline Arabidopsis 3 & 231.7 & 2.05 & 1.65 & 481.3 & 2.10 & 2.18 \\
\hline Tomato 1 & 404.7 & 2.11 & 2.06 & 286.0 & 2.00 & 2.07 \\
\hline Tomato 2 & 382.9 & 1.99 & 1.88 & 458.3 & 2.12 & 2.09 \\
\hline Tomato 3 & 340.0 & 2.11 & 1.95 & 592.3 & 2.07 & 2.16 \\
\hline Wheat 1 & 238.0 & 2.07 & 1.51 & 379.1 & 2.08 & 2.20 \\
\hline Wheat 2 & 341.5 & 2.12 & 1.40 & 581.1 & 2.15 & 2.33 \\
\hline Wheat 3 & 332.5 & 2.10 & 2.02 & 340.0 & 2.07 & 2.24 \\
\hline
\end{tabular}

RNA concentration and absorbance ratios ( $A_{260 / A 230}$ and $\left.A_{260 / A 280}\right)$ are indicated per sample and extraction method. Samples were extracted and analysed in triplicates, as indicated. Starting material per sample was as follows: Arabidopsis leaves - eight leaf discs ( $7 \mathrm{~mm}$ diameter) of five-week-old plants grown in short day conditions; wheat leaves - eight leaf discs (7 mm diameter) of four-week-old plants grown in the glasshouse; tomato leaves - eight leaf discs (7 mm diameter) of five-week-old plants grown in the glasshouse. Samples were resuspended in $30 \mu \mathrm{L}$ of water in all cases.

were obtained (Table 1, Additional file 2: Figure S1). Five hundred theoretical nanograms of RNA, according to the spectrophotometric quantification, were then loaded on a $1.5 \%$ agarose gel in TBE with in-gel ethidium bromide staining, alongside with an RNA ladder $(0.5-10 \mathrm{~Kb}$ RNA ladder, Life Technologies), in order to check the integrity of the isolated nucleic acids.

As shown in Table 1, in our hands, the theoretical concentration of RNA obtained from a given sample (Arabidopsis, tomato or wheat) was slightly higher when using the procotol by Oñate-Sánchez and VicenteCarbajosa [4], according to the spectrophotometric quantification. Additionally, nucleic acid purity was higher in the RNA samples obtained following this protocol: while the $A_{260 / A 230}$ and $A_{260 / A 280}$ ratios were above 2.0 for these samples, representing high purity, in all cases, the $A_{260 / A 230}$ value in the samples obtained using TRI reagent was generally lower (ranging between 1.40 and 2.06), indicating polysaccharide or, most likely, polyphenol contamination. Of note, it is known that traces of phenol contaminants can strongly inhibit downstream steps in genomic approaches, therefore compromising the results. Additionally, high absorbance at $\mathrm{A}_{230}$ can lead to the overestimation of RNA concentration in the sample.

Samples obtained from all three species using either protocol yielded RNA which was good quality (Figure 1). Of note, even though the same theoretical amount of RNA $(0.5 \mu \mathrm{g})$ was loaded in all lanes, samples obtained

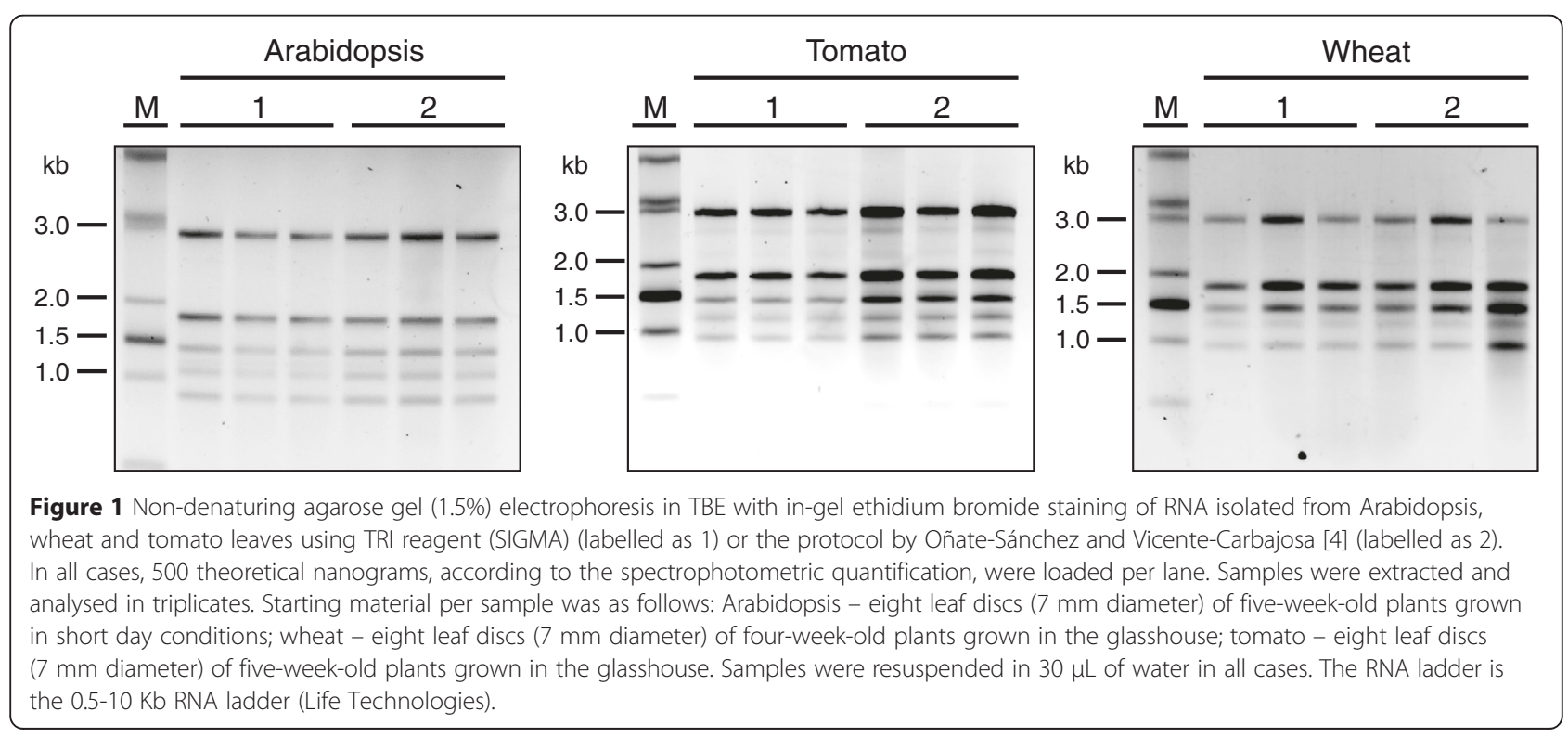


using the commercial reagent usually appeared slightly fainter, indicating an overestimation of the RNA concentration most likely due to high absorbance at $\mathrm{A}_{230}$.

Our results demonstrate that the improved RNA isolation protocol published by Oñate-Sánchez and VicenteCarbajosa [4] can be successfully applied to other plant species, including monocots, without further modification. Besides tomato and wheat, we have also successfully applied this method to Citrus reticulata, Citrus limon, Solanum tuberosum, Solanum americanum, Amaranthus viridis and Malva parviflora (Ana Arruabarrena, unpublished). Moreover, this protocol yields RNA whose quality is at least comparable to that provided by commercially available phenol-based reagents (Table 1, Figure 1), and the resulting nucleic acids can be directly used in downstream applications. Its simplicity and the low cost of materials used make this protocol widely accessible; therefore, it can be easily implemented in laboratories worldwide, allowing affordable and easy RNA isolation from different plant species.

\section{Additional files}

Additional file 1: Supplementary materials for plant RNA extraction protocol.

Additional file 2: Figure S1. Absorbance curves obtained for the samples shown in Figure 1 and Table 1. Method 1. TRI Reagent method; Method 2. Protocol from Oñate-Sánchez \& Vicente-Carbajosa (2008) [4].

\section{Competing interests}

The authors declare that they have no competing interests.

\section{Authors' contributions}

DC, LS, AA and RL-D designed and performed experiments. RL-D supervised the work. RL-D and CZ wrote the manuscript. All authors read and approved the final manuscript.

\section{Acknowledgements}

This work was supported by the Gatsby Charitable Foundation. DC is the recipient of a fellowship from the Portuguese Foundation for Science and Technology (FCT) (reference SFRH/BD/79088/2011). Research in the R.L-D.'s laboratory is supported by the Shanghai Center for Plant Stress Biology, Chinese Academy of Sciences.

\section{Author details}

${ }^{1}$ The Sainsbury Laboratory, Norwich Research Park, Norwich NR4 7UH, United Kingdom. ${ }^{2}$ National Agricultural Research Institute (INIA), Estación Experimental INIA Salto Grande, Salto CP50000, Uruguay. ${ }^{3}$ Shanghai Institutes of Biological Sciences, Shanghai Center for Plant Stress Biology (PSC),

Chinese Academy of Sciences, Shanghai 201602, China.

Received: 24 June 2014 Accepted: 1 April 2015

Published online: 16 April 2015

\section{References}

1. Carpenter CD, Simon AE. Preparation of RNA. Methods Mol Biol. 1998;82:85-9.

2. Chomczynski P. A reagent for the single-step simultaneous isolation of RNA, DNA and proteins from cell and tissue samples. Biotechniques. 1993;15(3):532-4. 536-7.

3. Weigel D, Glazebrook J. Arabidopsis: a laboratory manual. New York: Cold Spring Harbor: Cold Spring Harbor Laboratory Press; 2002.

4. Oñate-Sánchez L, Vicente-Carbajosa J. DNA-free RNA isolation protocols for Arabidopsis thaliana, including seeds and siliques. BMC Res Notes. 2008;1:93.

\section{Submit your next manuscript to BioMed Central and take full advantage of:}

- Convenient online submission

- Thorough peer review

- No space constraints or color figure charges

- Immediate publication on acceptance

- Inclusion in PubMed, CAS, Scopus and Google Scholar

- Research which is freely available for redistribution 